\title{
5-Aminolevulinic Acid Fluorescence in Detection of Peritoneal Metastases
}

\author{
Yutaka Yonemura ${ }^{1,2,3,4}$, Emel Canbay ${ }^{1,2,3}$, Haruaki Ishibashi ${ }^{1,2}$, Eisei Nishino ${ }^{5}$, \\ Yoshio Endou $^{6}$, Shouzou Sako ${ }^{1}$, Shun-Ichirou Ogura ${ }^{7}$
}

\begin{abstract}
Background: The value of 5-aminolevulinic acid (ALA) in fluorescence detection of peritoneal metastases and the underlying mechanisms were evaluated in patients with peritoneal surface malignancies. Materials and Methods: Oral 5-ALA was administered at a concentration of $20 \mathrm{mg} / \mathrm{kg}$ body weight with $50 \mathrm{ml}$ of water 2 hours prior to surgery $(n=115)$. The diagnostic value of 5-ALA based fluorescence production was evaluated following white light inspection during prior to cytoreductive surgery and hyperthermic intraperitoneal chemotherapy. Then, peptide transporter PEPT1 (ALA influx transporter) and ATP-binding cassette transporter ABCG2 (porphyrin efflux transporter) gene expression was determined with quantitative real time (qRT)-PCR and pathological diagnoses confirmed for all tissue samples. Results: The 5-ALA based photodynamic detection rate was $17 \%$ for appendiceal mucinous neoplasms, $54 \%$ for colorectal cancers, $33 \%$ for gastric cancers, $67 \%$ for diffuse malign peritoneal mesotheliomas, and $89 \%$ for epithelial ovarian cancer of peritoneal metastases. 5-ALA was detected in all cases of peritoneal metastases originating from cholangiocarcinomas whereas it was not able to detect any in granulosa cell and gastrointestinal stromal tumor cases. Furthermore, PEPT1 was overexpressed whereas ABCG2 expression was downregulated in tumors detected with fluorescence. Conclusions: 5-ALA provided $100 \%$ specificity and high sensitivity to detect peritoneal metastases in subgroups of patients with peritoneal surface mailgnancies. ALA influx transporter PEPT1 and porphyrin efflux transporter ABCG2 genes are important in tumor specific 5-ALA induced fluorescence in vivo. Further studies should clarify diagnostic utility of 5-ALA in peritoneal surface malignancies.
\end{abstract}

Keywords: Aminolevulinic acid - photodynamic diagnosis - peritoneal surface malignancies - PEPT1 - ABCG2

Asian Pac J Cancer Prev, 17 (4), 2271-2275

\section{Introduction}

Peritoneal involvement of gastrointestinal, gynecologic and mesothelial carcinomas are named as Peritoneal Surface Malignancies (PSM). Previous studies suggested that volume of peritoneal seeding has an impact on survival in PSM (Chu et al., 1989; Sadeghi et al., 2000; Jayne et al., 2002). The natural course of tumors at this stage is characterized by a short life expectancy (Sadeghi et al., 2000; Yan et al., 2007). Palliative systemic chemotherapy and best supportive care are widely used as treatment modalities. Surgery was performed in palliative intention to prevent tumor related complication as bleeding, obstruction or perforation. The options for management of PSM have been expanded over the past three decades. Sugarbaker introduced cytoreductive surgery (CRS) and hyperthermic intraperitoneal chemotherapy (HIPEC) as a new treatment modality for selected patients with
PSM (Sugarbaker et al., 1989; Sugarbaker, 2006). This multimodal approach is directed to surgically eliminate all the visible nodules, followed by intraoperative direct instillation of heated chemotherapy in to the abdominal cavity to eradicate the remaining microscopic tumoral foci and free cancer cells. Studies from different centres have investigated the outcome and utility of this combined treatment modality in these patients. All these studies show that completeness of cytoreduction and extent of disease are the most relevant predictors of survival (Glehen et al., 2004; Verwaal et al., 2008; Yan et al., 2009; Glehen et al., 2010; Yang et al., 2011; Chua et al., 2012), suggesting the complete cytoreductive surgery is still crucial in management of patients with peritoneal surface malignancies. Therefore, determination of tumoral foci invisible with white light inspection prior to complete cytoreduction and HIPEC is mandatory to provide better survival outcomes in patients with PSM.

${ }^{1}$ NPO Organization to Support Peritoneal Dissemination Treatment, Harukimotomachi, ${ }^{2}$ Department of General Surgery, ${ }^{5}$ Department of Pathology, Kishiwada Tokushukai Hospital, Osaka, ${ }^{4}$ Department of General Surgery, Kusatsu General Hospital, Yabase, ${ }^{7}$ Graduate School of Bioscience and Biotechnology, Tokyo Institute of Technology, Central Research Resource Branch, Cancer Research Institute, Kanazawa University, Yokohama, Japan, ${ }^{3}$ NPO HIPEC Istanbul, Istanbul, Turkey, ${ }^{6}{ }^{*}$ For correspondence: drecanbay@gmail.com 
Diagnosis of PSM is challenging especially when the lesions are flat or small enough avoiding detection with preoperative investigations. The recent interest in this field has resulted new techniques that enable better identification of tumor extension into the abdominal cavity to improve diagnosis of peritoneal seeding in PSM. Initially, photodynamic diagnosis (PDD) has been proposed for PSM in animal models (Canis et al., 2001;Krammer and Plaetzer, 2008). The identification of peritoneal metastases was significantly increased by 5-aminolevulinic acid (ALA) administration followed by fluorescence detection in these studies. ALA is the natural precursor of the heme pathway. ALA induced protophorphyrin (Pp)IX accumulation from cancer cells has been applied as a red fluorescence detection marker of cancer using fluorescence blue light (Krammer and Plaetzer, 2008). More recently, PDD is used for detection of PSM originated from gastric cancer using laparoscopic method (Kishi et al., 2012). We previously reported PDD of peritoneal metastatic nodules in primary peritoneal papillary serous carcinoma as a case report after oral administration of 5-ALA (Canbay et al., 2014) and pivotal roles of peptide transporter PEPT1 (ALA influx transporter) and ATP-binding cassette (ABC) transporter ABCG2 (porphyrin efflux transporter) genes in accumulation of PpIX and 5-ALA induced photocytotoxicity of gastric cancer cells in vitro (Hagiya et al., 2012). Now, we present results of our prospective study. We aim to explore the role of 5-ALA in detection of peritoneal metastases and the mechanism underlying photosensitivity of metastatic nodules in vivo.

\section{Materials and Methods}

One hundred fifteen patients admitted to NPO Organization to Support Peritoneal Dissemination Treatment, Osaka, Japan with a clinical diagnosis of PSM included to this study. The local ethics committee in our hospital approved the current study (Ethical Committee of Kishiwada Tokushukai Hospital (No-2405). A written informed consent was obtained from all patients. Exclusion criteria were extra abdominal and liver metastases.

\section{Fluorescence Detection of Peritoneal Metastases}

Each patient received $20 \mathrm{mg} / \mathrm{kg}$ body weight of 5-ALA (Cosmo Bio Co., Ltd, Tokyo, Japan) dissolved in $50 \mathrm{ml}$ water, then mixed with $50-100 \mathrm{ml}$ orange juice. The mixture was given orally 4 hours before surgery. After oral administration, patients were kept away from direct sunlight or strong room light for 24 hours. Following standard evaluation under the standard white light, PDD was performed using a xenon lamp (300W) with blue light with a 375-445nm wavelength to detect fluorescence. Excision of tumor nodules from peritoneal surfaces was performed and subjected to histological examination and molecular analysis. Then cytoreductive surgery (CRS) with peritonectomy and hyperthermic intraperitoneal chemotherapy (HIPEC) were performed in all patients with PSM as described previously by Sugarbaker (1998).

Detection of PEPT1 and ABCG2 expression levels
Total RNA was extracted from tissue samples, and first- strand cDNA was prepared from total RNA by RT reaction, as described previously (Hagiya et al., 2008). Thereafter, the first-strand cDNA preparations encoding PEPT1, ABCG2, and GAPDH were individually amplified by PCR in a Thermal Cycler Dice Mini (TaKaRa Bio, Otsu, Japan) with specific primer sets as previously reported (Hagiya et al., 2012). GAPDH is used as the internal control. The PCR reaction consisted of hot-start incubation at $95^{\circ} \mathrm{C}$ for $5 \mathrm{~min}$ and 35 cycles each of $95^{\circ} \mathrm{C}$ for $30 \mathrm{~s}$ and $60^{\circ} \mathrm{C}$ for $1 \mathrm{~min}$. The resulting amplicons were separated by $2.5 \%$ agarose gel electrophoresis and detected with ethidium bromide under ultraviolet light.

\section{Immunoblot analyses}

Immunoblot analyses were carried out as previously described (Hagiya et al., 2008). We used H-235 specific to human PEPT1 polyclonal antibody (1:200 dilution; Santa Cruz Biotechnology, Santa Cruz, CA, USA), BXP-21 specific to human ABCG2- monoclonal antibody (1:200 dilution; Convance Research Products, Emeryville, CA), and rabbit muscle GAPDH monoclonal antibody (1:1000 dilution; American Research Products, Belmont, MA) as a first antibody. Anti-mouse IgG HRP-conjugated antibody (Cell Signaling Technology, Beverly, MA, USA) and antirabbit IgG HRP conjugates (Santa Cruz Biotechnology) at 1:3000 dilutions were used as secondary antibodies.

\section{Statistical analysis}

Data were collected for all patients included in the study. Microsoft excel 10.0 was used to manage and analyse the data. For statistical analysis, the SPSS version
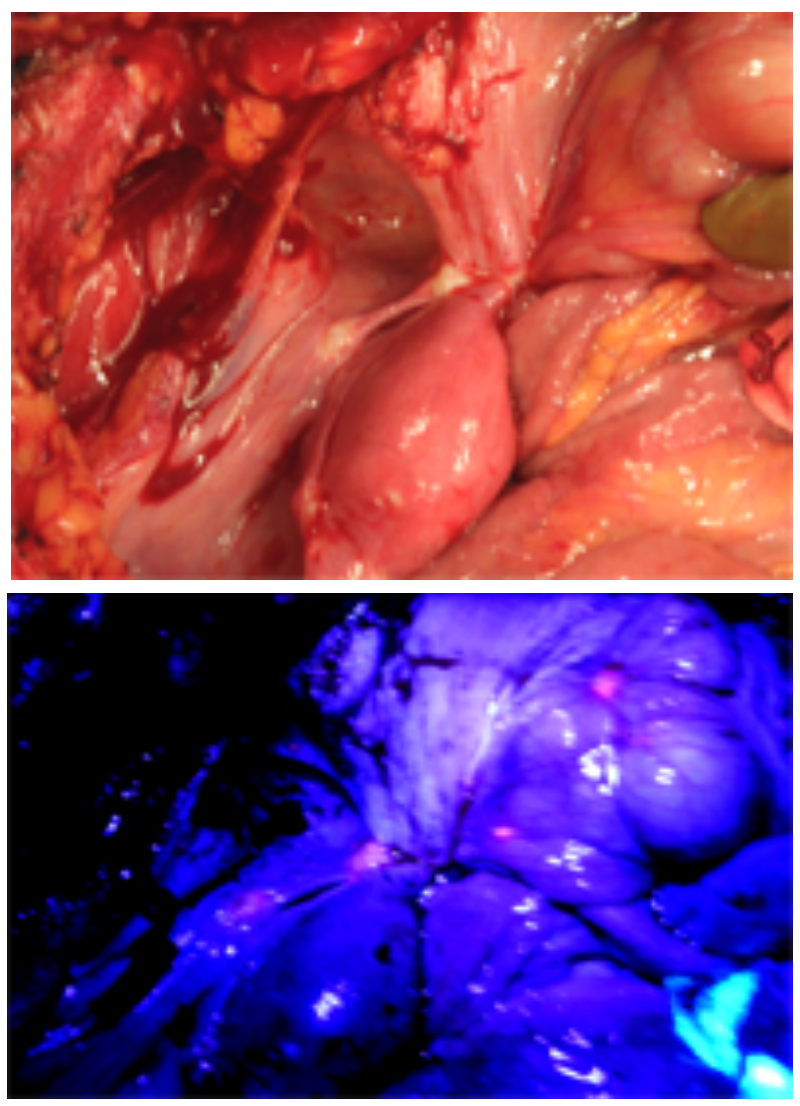

Figure 1. White Light and Photodynamic Detection of Peritoneal Metastatic Nodules in Patients with PSM 
17.0 statistical software package (SPSS, Chicago, Ill) was used.

\section{Results}

\section{Patient information}

One hundred eighteen patients with PSM included in the study (with a mean age of $63.0 \pm 11$ and a male to female ratio of 49:69). Histopathological diagnosis included peritoneal metastases from appendiceal mucinous adenocarcinoma $(n=48)$, cholangiocarcinoma $(n=4)$, colorectal adenocarcinoma $(n=17)$, gastric adenocarcinoma (24), granulosa cell tumor $(n=3)$, Gastrointestinal stromal tumor of small bowel GIST $(n=4)$, diffuse malign peritoneal mesothelioma $(n=6)$, ovarian/ extra ovarian primary peritoneal carcinoma $(n=9)$.

\section{Diagnostic value of 5-ALA in PSM}

A typical observation of metastatic nodules with sequence of white light and PDD is shown in Figure 1. About $0.5 \mathrm{~mm}$ tumor nodules were detected by PpIX fluorescence (Figure 2). Overall metastases detected 42 out of 118 patients with PSM (Table 1). Even though, there was no significant improvement in visualization of peritoneal tumor lesions, PDD was allowed enhanced detection of metastatic nodules of cholangiocarcinoma (100\%), ovarian/extra ovarian primary peritoneal cancer (89\%), and malign mesothelioma (67\%). PDD detection rate of tumor nodules from colorectal adenocarcinoma was $53 \%$, pancreas adenocarcinoma was $50 \%$, from gastric and

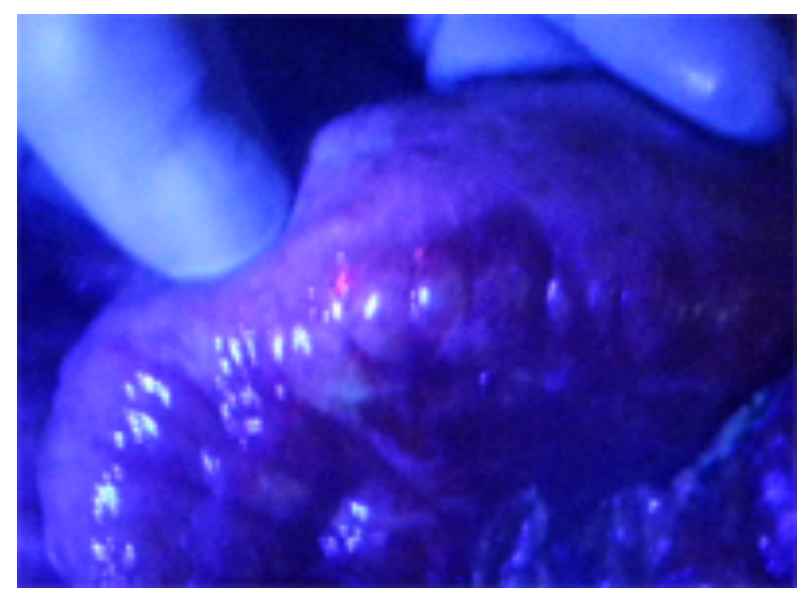

Figure 2. Metastatic Tumor Foci Less than $0.5 \mathrm{~cm}$ Can be Detected with Fluorescence small bowel adenocarcinoma were $33 \%$, and appendiceal mucinous adenocarcinoma was $17 \%$. Although very limited number of PSM patients with granulosa cell tumor $(n=3)$ and GIST $(n=4)$ investigated, tumor nodules were not visualized with PDD in both groups of patients.

Nausea $(n=1)$ and vomiting $(n=1)$ as adverse effects following 5-ALA administration were observed in 2 patients $(1,6 \%)$. Direct exposure of sunlight or strong room light was avoided for 24 hour after operation. Symptoms of porphyria were not observed in any of our patients group.

Characterization of porphyrin biosynthesis pathwayrelated genes PEPT1 and ABCG2 expression levels in 5-ALA-PDD positive specimens of patients with PSM

We examined the expressions of PEPT1 and ABCG2 genes involved in the porphyrin biosynthesis pathway in tumor-specific PpIX accumulation following the administration of 5-ALA in vivo in clinical specimens

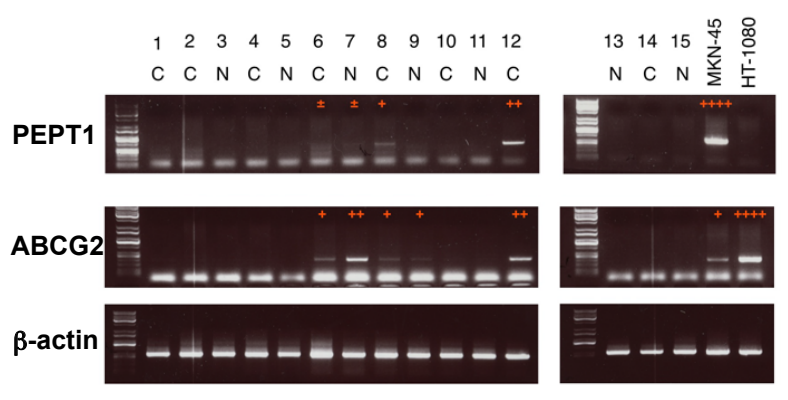

Fluorescence

Emission

Figure 3. mRNA Expression of PEPT1 and ABCG2 Genes in Peritoneal Metastatic Nodules Detected with Fluorescence

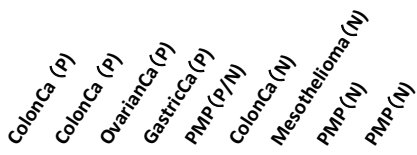

Peptide transporter (PEPT1)

ATP-binding cassette transporter (ABCG2)

Figure 4. PEPT1 and ABCG2 Protein Levels with Fluorescence Using Western Blotting in PSM Originated from Different Malignancies. PEPT1 protein expression was upregulated whereas ABCG2 protein expression was downregulated in peritoneal metastatic nodules detected with fluorescence

Table 1. Peritoneal Metastatic Nodule Detection with 5-ALA Induced Fluorescence

\begin{tabular}{|c|c|c|c|c|c|c|c|}
\hline PSM & Pts detected with 5-ALA & Sensitivity & Specificity & NLR & $95 \% \mathrm{CI}$ & PPV & NPV \\
\hline & $(n=115)$ & $(\%)$ & $(\%)$ & $(\%)$ & $(\%)$ & $(\%)$ & $(\%)$ \\
\hline Appendiceal Cancer & $8 / 48$ & 17 & 100 & 0.83 & $(0.73-0.95)$ & 100 & 54.5 \\
\hline Cholangiocarcinoma & $2 / 2$ & 100 & 100 & 0 & - & 100 & 100 \\
\hline Colorectal cancer & $9 / 17$ & 53 & 100 & 0.47 & $(0.28-0.78)$ & 100 & 68 \\
\hline Gastric Cancer & $8 / 24$ & 33 & 100 & 0.67 & $(0.5-0.88)$ & 100 & 60 \\
\hline Granulosa cell tumor & $0 / 3$ & 0 & 100 & 1 & $(1.0-1.0)$ & - & 50 \\
\hline GIST & $0 / 1$ & 0 & 100 & 1 & $(1.0-1.0)$ & - & 50 \\
\hline Malign mesothelioma & $4 / 6$ & 66 & 100 & 0.33 & $(0.11-1.03)$ & 100 & 75 \\
\hline Epithelial ovarian cancer & $8 / 9$ & 89 & 100 & 0.11 & $(0.02-0.71)$ & 100 & 90 \\
\hline
\end{tabular}

NLR, negative likelihood ratio; PPV, positive predicting value; NPV, negative predicting value 
Yutaka Yonemura et al

of patients with PSM. The mRNA levels of PEPT1 and ABCG2 were measured as shown in Figure 3 and expressions were evaluated relative to those of $\beta$-actin expression. PEPT1 mRNA levels were upregulated whereas ABCG2 mRNA levels were downregulated in tumor tissues of PSM patients detected with 5-ALA-PDD $(p<0.05$; Figure 3$)$. These results suggest that high PEPT1 expression and low ABCG2 expression play pivotal role in tumor specific PpIX accumulation following 5-ALA administration in PDD of PSM patients.

PEPT1 and ABCG2 protein expressions in 5-ALA-PDD positive specimens of patients with PSM

We further determined the PEPT1 and ABCG2 protein expression levels in tumor tissue samples from PSM. We detected PEPT1 protein expression is upregulated and ABCG2 protein expression is downregulated in tumor tissues detected with 5-ALA-PDD ( $\mathrm{p}<0.05$; Figure 4$)$.

\section{Discussion}

This study provided promising results in detection of metastatic nodules with 5-ALA induced photodynamic method in selected patients with PSM. Even though overall detection rate was $35.6 \%$, PDD was allowed enhanced detection of metastatic nodules in all cholangiocarcinoma patients, in majority of ovarian/extra ovarian primary peritoneal cancer patients (89\%) and malign mesothelioma patients $(67 \%)$. In addition to this, metastatic tumor nodules could be able to detect in PSM patients originated from colorectal adenocarcinoma (53\%), from gastric adenocarcinoma (33\%), and appendiceal mucinous adenocarcinoma (17\%). On the other hand, PDD was not effective in diagnosis of metastatic peritoneal nodules in PSM patients with granulosa cell tumor and GIST. Although different determination ratio was obtained in PSM patients, sensitivity of this method was $100 \%$ if metastases could be able to detect with PDD.

Furthermore, upregulation of PEPT1 (ALA influx transporter) and downregulation of ABCG2 (porphyrin efflux transporter) genes could play pivotal role as an underlying mechanism in 5-ALA induced tumor specific PpIX accumulation for photodynamic diagnosis of peritoneal metastatic nodules in patients with PSM.

5-ALA has approved as a photosensitizer of PDD (Ladner et al., 2001). ALA-PDD guided surgery has improved surgical results and recurrence-free survival rates in patients with malignant glioma (Stummer et al., 2006). Moreover, the introduction of 5-ALA with cystoscopy for early bladder cancer (Daniltchenko et al., 2005; Denzinger et al., 2007) and urinary tract transitional cell carcinoma (Ahmad S et al., 2012) has increased the number of recurrence-free patients and improved recurrence-free survival after five years compared to white-light cystoscopy.

For peritoneal metastasis, intraperitoneal application of 5-ALA improved the sensitivity of peritoneal metastasis identification in ovarian cancer (Loning et al., 2004). Oral 5-ALA administration has improved the detection of peritoneal metastases in patients with pancreatic adenocarcinoma (Orth et al., 2000). Our results from ovarian carcinoma, pancreas adenocarcinoma and cholangiocarcinoma are similar with these previous studies.

5-ALA-PDD improved the detection sensitivity over white-light examination during staging laparoscopy in patients with peritoneal carcinomatosis originated from gastrointestinal cancers (Zopf et al., 2005) and gastric cancer (Hagiya et al., 2012; Zopf et al., 2005). Finally, they conclude that 5-ALA-PDD yielded more lesions detected than the conventional white-light examination. In our study, 5-ALA-PDD also improved the determination of small metastatic nodules less than $0.5 \mathrm{~mm}$ in patients with PSM. A recent systematic review analyzed 12 human and 18 animal studies (Almerie et al., 2015). They have found that PDD was safe with no significant adverse effects. Detection of malignant peritoneal nodules was increased by $21 \%-34 \%$ in comparison with white light alone. The sensitivity and specificity of PDD were reported at $83 \%$ $100 \%$ and $95 \%-100 \%$, respectively. These findings were also supported by multiple animal studies, which have shown an increase in the sensitivity of tumor detection when using PDD (72\%-91\%) in comparison with white light alone (39\%).

In this study, we investigated the roles of PEPT1 and ABCG2 in 5-ALA induced PpIX accumulation in vivo. Our results strongly suggest that PSM share a common mechanism with previously reported in vitro results for tumor-specific PpIX accumulation following the administration of 5-ALA.

There are some limitations of this study; first of all, we had very limited sample size in some subgroups of peritoneal metastases. Specially, sample size for peritoneal metastases of cholangiocarcinoma, granulosa cell tumor and GIST were very limited due to natural occurrence of these disease are rare even metastases are the most rare to complete significant sample size. Another limitation, we only detect the samples in patients with peritoneal metastases. Comparison of diagnostic value of 5-ALA between diseases and their peritoneal metastases as well as other diseases needs to be performed to clarify exact role of 5-ALA based fluorescence production in detection of metastases.

In conclusion, 5-ALA guided surgery can be performed safely with high detection rate of peritoneal implants around 5mm, in subgroups of PSM patients originated from common bile duct and ovarian/extra ovarian primary peritoneal carcinoma compared to those of originated from colorectal, stomach, primary peritoneal malign mesothelial origin and appendiceal mucinous neoplasms to achieve complete cytoreduction. Continued research will clarify whether this diagnostic intervention will be altered the prognosis and therapeutic approaches of PSM.

\section{Acknowledgements}

Authors declare that their no financial interest for this research. We are grateful to Professor Bjorn L.D.M. Brücher for his critical reading of the text and for his critical comments. 


\section{References}

Ahmad S, Aboumarzouk O, Somani B, et al (2015). . Oral 5 -aminolevulinic acid in simultaneous photodynamic diagnosis of upper and lower urinary tract transitional cell carcinoma - a prospective audit. BJU Int, 110, 596-600

Almerie MQ, Gossedge G, Wright KE, et al (2015). . Photodynamic diagnosis for detection of peritoneal carcinomatosis. J Surg Res, 195, 175-87

Canbay E, Ishibashi H, Sako S, et al (2014). Photodynamic detection and management of intraperitoneal spreading of primary peritoneal papillary serous carcinoma in a man: report of a case. Surg Today, 44, 373-7

Canis M, Botchorishvili R, Berreni N, et al (2001). 5-Aminolevulinic acid-induced (ALA). fluorescence for the laparoscopic diagnosis of peritoneal metastasis. AST An experimental study. Surg Endosc, 15, 1184-6

Chu DZ, Lang NP, Thompson C, et al (2004). Peritoneal carcinomatosis in nongynecologic malignancy. A prospective study of prognostic factors. Cancer, 63, 364-7

Chua TC, Moran BJ, Sugarbaker PH, et al (2012). Early- and long-term outcome data of patients with pseudomyxoma peritonei from appendiceal origin treated by a strategy of cytoreductive surgery and hyperthermic intraperitoneal chemotherapy. J Clin Oncol, 30, 2449-56

Daniltchenko DI, Riedl CR, Sachs MD, et al (2005). Longterm benefit of 5-aminolevulinic acid fluorescence assisted transurethral resection of superficial bladder cancer: 5-year results of a prospective randomized study. J Urol, 174, 2129-33

Denzinger S, Burger M, Walter B, et al (2007). Clinically relevant reduction in risk of recurrence of superficial bladder cancer using 5-aminolevulinic acid-induced fluorescence diagnosis: 8- year results of prospective randomized study. Urol, 69, 675-9

Glehen O, Cotte E, Schreiber V, et al (2004). Intraperitoneal chemohyperthermia and attempted cytoreductive surgery in patients with peritoneal carcinomatosis of colorectal origin. Br J Surg, 91,747-54

Glehen O, Gilly FN, Arvieux C, et al (2010). Association Française de Chirurgie Peritoneal carcinomatosis from gastric cancer: a multi-institutional study of 159 patients treated by cytoreductive surgery combined with perioperative intraperitoneal chemotherapy. Ann Surg Oncol, 17, 2370-7

Hagiya Y, Adachi T, Ogura S, An R, et al (2008). Nrf2dependent induction of human ABC transporter ABCG2 and heme oxygenase- 1 in HepG2 cells by photoactivation of porphyrins: biochemical implicationsfor cancer cell response to photodynamic therapy. J ExpTher Oncol, 7, 153-67

Hagiya Y, Endo Y, Yonemura Y, et al (2012). Pivotal roles of peptide transporter PEPT1 and ATP-binding cassette (ABC), transporter ABCG2 in 5-aminolevulinic acid (ALA). -based photocytotoxicity of gastric cancer cells in vitro. Photodiagnosis Photodynamic Therapy, 9, 204-14.

Jayne DG, Fook S, Loi C, et al (2002). Peritoneal carcinomatosis from colorectal cancer. Br J Surg, 89,1545-50

Kishi K, Fujiwara Y, Yano M, et al (2012). Staging laparoscopy using ALA-mediated photodynamic diagnosis improves the detection of peritoneal metastases in advanced gastric cancer. J Surg Oncol, 106, 294-8

Krammer B and Plaetzer K. (2008). ALA and its clinical impact, from bench to bedside. Photochem Photobiol Sci, 7, 283-9

Ladner DP, Steiner RA, Allemann J, et al (2001). Photodynamic diagnosis of breast tumors after oral application of aminolevulinic acid. Br J Cancer, 84, 33-7

Loning M, Diddens H, Küpker W, et al (2004). Laparoscopic fluorescence detection of ovarian carcinoma metastases using 5-aminolevulinic acid-induced protoporphyrin IX. Cancer, 100, 1650-6

Orth K, Russ D, Steiner R, et al (2000). Fluorescence detection of small gastrointestinal tumours: Principles, technique, first clinical experience. Langenbecks Arch Surg, 385,488-94

Sadeghi B, Arvieux C, Glehen O, et al (2000). Peritoneal carcinomatosis from non- gynecologic malignancies: results of the EVOCAPE 1 multicentric prospective study. Cancer, $\mathbf{8 8}, 358-63$.

Stummer W, Pichlmeier U, Meinel T, et al (2006). ALAGlioma Study Group. Fluorescence-guided surgery with 5-aminolevulinic acid for resection of malignant glioma: a randomised controlled multicentre phase III trial. Lancet Oncol, 7, 392-401

Sugarbaker PH, Cunliffe WJ, Belliveau J, et al (1989). Rationale for integrating early postoperative intraperitoneal chemotherapy into the surgical treatment of gastrointestinal cancer. Semin Oncol, 16, 83-97

Sugarbaker PH (1998). Intraperitoneal chemotherapy and cytoreductive surgery for the prevention and treatment of peritoneal carcinomatosis and sarcomatosis. Semin Surg Oncol, 14, 254-61

Sugarbaker PH (2006). New standard of care for appendiceal epithelial neoplasms and pseudomyxoma peritonei syndrome? Lancet Oncol, 7, 69-76

Verwaal VJ, Bruin S, Boot H, et al (2008). 8-year followup of randomized trial: cytoreduction and hyperthermic intraperitoneal chemotherapy versus systemic chemotherapy in patients with peritoneal carcinomatosis of colorectal cancer. Ann Surg Oncol, 15, 2426-32

Yan TD, Brun EA, Cerruto CA, et al (2007). Prognostic indicators for patients undergoing cytoreductive surgery and perioperative intraperitoneal chemotherapy for diffuse malignant peritoneal mesothelioma. Ann Surg Oncol, 14, 41-9

Yan TD, Deraco M, Baratti D, et al(2009). Cytoreductive surgery and hyperthermic intraperitoneal chemotherapy for malignant peri toneal mesothelioma: multi-institutional experience. J Clin Oncol, 27, 6237-42

Yang XJ, Huang CQ, Suo T, et al (2011). Cytoreductive surgery and hyperthermic intraperitoneal chemotherapy improves survival of patients with peritoneal carcinomatosis from gastric cancer: final results of a phase III randomized clinical trial. Ann Surg Oncol, 18, 1575-81

Zopf T, Schneider AR, Weickert U, et al (2005). Improved preoperative tumor staging by 5 -aminolevulinic acid induced fluorescence laparoscopy. Gastrointest Endosc, 62,763-7 